\title{
Available Link Bandwidth Based Network Selection in Multi-access Networks
}

\author{
Kiran Ahuja \\ Dept. of Electronics and Communication Engineering, DAV Institute of Engg \& Technology, Jalandhar, Punjab, India \\ E-mail: askahuja2002@yahoo.co.in \\ Brahmjit Singh, Prof. \\ Dept. of Electronics and Communication Engineering, National Institute of Technology, Kurukshetra, Haryana, India \\ E-mail: brahmjit.s@gmail.com
}

Rajesh Khanna, Prof.

Electronics and Communication Engineering Department, Thapar University, Patiala, Punjab, India E-mail: rkhanna@thapar.edu

\begin{abstract}
In a heterogeneous wireless environment, one of the important aspects of seamless communication for ubiquitous computing is the dynamic selection of the best access network. The problem of access network selection has been addressed through various decision methods based on available network information. Available link bandwidth is one of the important information parameters, which can be used as criterion for network selection. In this paper, we consider available bandwidth as a dynamic parameter to select the network in heterogeneous environment. First, we propose a bootstrap approximation based technique to estimate available bandwidth and then utilize it for the selection of the best suitable network in the heterogeneous environment consisting of $2 \mathrm{G}$ and $3 \mathrm{G}$ standards based wireless networks. The proposed algorithm is implemented in temporal and spatial domains to check its robustness. Estimation time with varying size of files is used as the performance metric. Through numerical results, it is shown that the proposed algorith $\mathrm{m}$ gives improved performance as compared to the existing algorithm.
\end{abstract}

Index Terms - 2G, 3G, Heterogeneous Networks, Bootstrap Approximation, Available Bandwidth

\section{Introduction}

There has been tremendous progress in the development and deployment of wireless technologies in the last decade. The ubiquitous computing and communication environment is also provided by next generation wireless networks. In order to realize the concept of 'Always Best Connected' (ABC) network, it is imperative to achieve seamless communication in heterogeneous environment consisting of diverse access technologies. To achieve this goal, one of the important problems in next generation wireless networks is to select the best available network meeting the quality of service require ments of the user as well. Available link bandwidth is one of the basic parameters to support a given service/application and hence ensures the quality of service required as the user moves from one access network to another.

The existing deployment of wireless technologies contains both $2 \mathrm{G}$ and $3 \mathrm{G}$ standards. Network selection in such heterogeneous environment is an important research problem. In this paper, we aim to present an available link bandwidth based network selection algorithm. Available bandwidth estimation is derived through bootstrap approximation method, which is then applied to select the appropriate network in heterogeneous environment consisting of $2 \mathrm{G}$ and $3 \mathrm{G}$ standards. 2G standard, for example, GSM (Global System for Mobile Communication) and 3G standardHSDPA (High Speed Downlink Packet Access) wireless technologies typically provide data access rate which may vary from $9.6 \mathrm{Kbps}$ through $2 \mathrm{Mbps}$ for $3 \mathrm{G}$ [1]. These rates are generally adequate for services employing a low to medium bandwidth e.g. voice communications, text messaging, instant messaging, electronic mail with no or relatively s mall attachments etc. Both $2 \mathrm{G}$ and $3 \mathrm{G}$ are merely milestones in mobile technology and represent two different phases. 3G mobile networks have new set of communication protocols due to rapid increase in data services and development in hardware and software. It makes available many more features for mobile users such as internet, mobile $\mathrm{TV}$, video calls, video conferencing, mobile gaming whereas no such features are there in $2 \mathrm{G}$ [2]. There is only voice transfer in $2 \mathrm{G}, 3 \mathrm{G}$ allows for data transfer in addition to transmission of voice. The clarity of voice in $3 \mathrm{G}$ is significantly more than $2 \mathrm{G}$, and there are very little disturbances. It is a much more secure technology than 2G. As the commercial companies compete with each other to increase their 
market potential, so to save $2 \mathrm{G}$ customers and to bring a new $3 \mathrm{G}$ customers, there should be ways, to do that, the subscriber can move seamlessly from one cell to another cell covered by different networks, the customer actually will be unaware of underlying technology [3].

To access wireless network, the mobile terminal cannot dynamically select the best link due to the absence of network parameters, which may reduce the performance of the system. In this paper we propose an effective and efficient network selection scheme based on available link bandwidth estimation by using bootstrap approximation method based on the real time network state. Proposed algorithm allows mobile terminals to select the most appropriate network dynamically between $2 \mathrm{G} / 3 \mathrm{G}$ as per requisition of the user. $2 \mathrm{G}$ and $3 \mathrm{G}$ networks both have similar properties to transfer of data over mobile networks but differ in terms of speed and architecture. So, the choice between $2 \mathrm{G}$ or $3 \mathrm{G}$ networks have significant role for multimed ia services.

The rest of the paper is organized in this manner: Section II elaborates the related literature survey. Section III describes network selection algorith m under consideration. Experimental setup is discussed in section IV. Further describes the performance metrics used for network selection. Numerical results of performance metrics are discussed in Section V. Finally, results are concluded in Section VI.

\section{Related Work}

Service continuity in heterogeneous wireless access technologies is a main issue. The challenge is to preserve continuous services while moving between these technologies. Appropriate selection of the network in heterogeneous environment is one of the main key. In this paper, we propose a network selection in heterogeneous environment based on available bandwidth estimation by using bootstrap approximation. When there are various types of probing techniques available for bandwidth estimation in literature [4-13]. An ideal probing tool provided accurate estimate of available bandwidth prior to sending data packets [4]. In the PATHON model there is no need to probe knowledge and management control over the network to provide an end to end measurement of available bandwidth. Further, it considers less traffic with least time for measurement [5]. The utilization of network connection is measured by using tri packets method defined in probing technique [6]. Packet pair probing technique is used by wireless network for bandwidth estimation while considering the capacity and the cross traffic variation of wireless link [7]. The probe gap model was designed for both one-hop and multi hop path, under the case of path persistent cross traffic. Further by adjusting the input-probing rate, bandwidth adaptive method was introduced [8].
An active probing tool was defined in [9], it was based on the concept of self-induced congestion, which run inside a real-time operating system and used denoising techniques to improve the measurement process Path quick method was also designed for quick end to end available bandwidth estimation. In this quick estimation was achieved by using a probing packet train [10]. Another available bandwidth estimation scheme was designed in which bandwidth availability was expressed in terms of a service curve. In this estimation was based on a sequence of probing packets [11]. A probabilistic methodology was also designed to estimate available bandwidth under non busy assumption and it also considered distribution of output probe gap [12]. A one-way delay jitter based scheme was developed for available bandwidth estimation that quantified the captured traffic ratio and identified the relationship between probing rate and available bandwidth [13]. Above discussed techniques introduce high additional traffic to the network which increase overhead, reduce reliability and due to these the estimation process become slow.

An available bandwidth measurement method for video streaming was defined in [14], in which difference of packet send times and feedbacks receive times was considered for estimation. Available bandwidth under bursty arrivals of discrete cross-traffic packets was estimated by queuing-theoretic foundation approach of single-hop packet-train [15]. In Minimal backlogging method, the available bandwidth of a queuing system was estimated; in this probing packets were sent to the queuing system by the nominal backlogging method [16]. Reliable available bandwidth estimation method distinguishes queuing regions which resolved false estimations [17]. The lightweight probing method estimated available bandwidth using queuing analysis approach in which investigation on Squared Coefficient of Variation (SCV) of the inter-departure process was made with periodic probing stream [18]. Queuing model described the mechanis $m$ for available bandwidth estimation using the output packet-pair dispersions [19]. One-way delay model was used to estimate available bandwidth in which proportional share of link capacity concept improved the measurement latency and accuracy [20]. The proposed algorithm in [21] selects network on the basis of available bandwidth estimation. It estimates the available link bandwidth on the basis of averaging of current sample only. Analysis revealed that proposed method effectively choose the suitable network for multimedia service by negotiating among available networks (i.e. WiMAX and $3 \mathrm{G}$ ).

Current available bandwidth estimation methods have been proposed by different authors. However, the network scenarios and metrics used in the evaluations are limited and their analysis about the applicability of the tools in real network applications is absent. An additional issue is that these evaluations do not include the amount of experiments needed to provide statistically valid conclusions. 
While in this paper, we propose an algorithm for network selection in heterogeneous environment of $2 \mathrm{G}$ \& $3 \mathrm{G}$ based on dynamic parameter i.e. available link bandwidth estimated by bootstrap approximation method to resolve the problem of network selection. Bootstrapping allows gathering many alternative versions of the single statistic that would ordinarily be calculated from one sample. So it is able to provide accurate and more reliable available link bandwidth estimation in comparison of existing techniques for network selection in wireless heterogeneous environment. The proposed network selection technique is described in following section.

\section{Proposed Network Selection Algorithm}

A novel network selection scheme is proposed in order to select the always best connected network in a given heterogeneous environment. The flow graph in Fig. 1 represents always best connected network in heterogeneous environment for multimedia services. The logic of network selection based on available bandwidth estimation with bootstrap approximation method and received signal strength (RSS) technique. Number of available wireless networks is possible because the user have multimode mobile device. In this paper, we assume that multimode device can access $2 \mathrm{G}$ and $3 \mathrm{G}$ networks. The selection of access network depends upon the user inclination that means on the application presently running. For multimedia services the foremost requirement is bandwidth. Access network selection depends on the network having more available bandwidth.

Step 1 Make sure presence of networks and then disconnect all available networks. Select one of the available networks and check its received signal strength. The performance of a wireless network connection depends partially on signal strength. The wireless signal strength in each direction determines the total amount of network bandwidth available along that connection. Received signal strength (RSS) is a measurement of the power present in a received radio signal. In general, the received signal $\mathrm{rt}$ at the time instant $t$ can be expressed as [22]:

$$
r t=a t s t-\tau+v t
$$

Here, $s$ denotes the transmitted (pilot) signal waveforms, at is the radio link attenuation, $\tau$ is the distance-dependent delay and $\mathrm{vt}$ is a noise component.

Step 2 Available bandwidth (ABW) is used as dynamic parameter for network selection in heterogeneous environment. The available bandwidth at a link is its unused capacity. Since, at any time, a link is either idle or transmitting packets at the maximum speed, the definition of the available bandwidth ought to look at the average unused bandwidth over some time interval $\mathrm{T}$.

$$
A_{\mathrm{i}}(t, T)=\frac{1}{T} \int_{t}^{T+t}\left(C_{\mathrm{i}}-\alpha_{\mathrm{i}}(t)\right)
$$

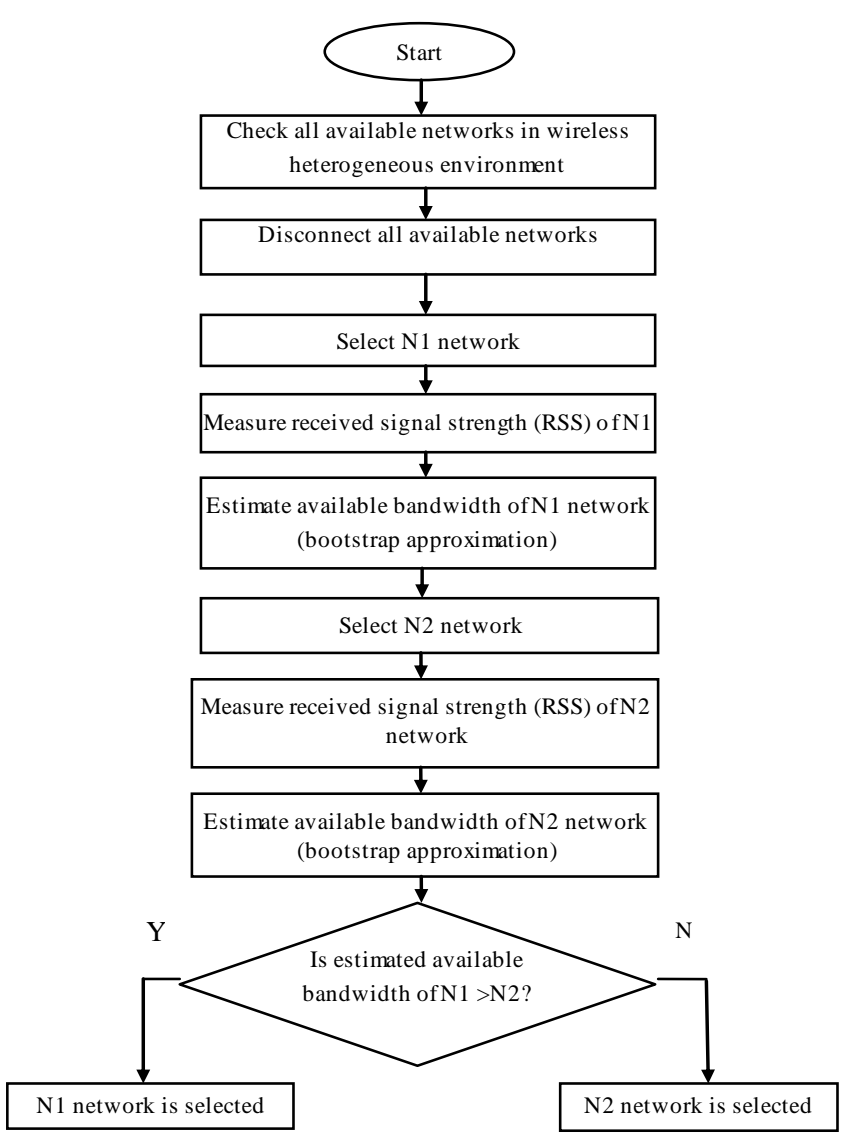

Fig. 1: Represents the flow chart of available bandwidth estimation algorithm for network selection in heterogeneous wireless environment

Where $A_{i}(t, T)$ is the available bandwidth at link $i$ at time $t, \lambda_{i}$ is its traffic and $C_{i}$ is the link's capacity. The available bandwidth along a link is measurement of minimu mavailable bandwidth of all traversed links [23]

Available Bandwidth in general, is non-stationary QoS information of wireless network. But when it is observed over a short time, it can be considered as stationary. In proposed algorithm, bootstrap approximation is employed to estimate available bandwidth by calculating the short-term stationary component.

Bootstrap Approximation method for available bandwidth estimation: $2 \mathrm{G} / 3 \mathrm{G}$ network's average available bandwidth is denoted by $\theta$ from a population with distribution function $(F)$, and then measured $2 \mathrm{G} / 3 \mathrm{G}$ available bandwidth. It is approximated during data acquisition window. Bootstrap method is considered to draw random samples $x_{i}$ from an unknown distribution function $(F)$ to form the new data set based on original data:

$$
X=\left(x_{1}, x_{2}, \ldots \ldots \ldots ., x_{n}\right)
$$


An original data set provides a simple estimate of the entire population by using discrete empirical distribution $(\mathrm{Fe})$. Each random sample $(\mathrm{x})$ has probability of $1 / n$, which has an equal likelihood of being chosen when re-sampling fro $\mathrm{m} F$ e. The bootstrap data set is then defined to be a random sample of size $n$ drawn with replacement from Fe. Since we want to estimate the parameter $\theta$ e by calculating a statistic from a random sample, correspondingly, we can calculate the same statistic from a bootstrap data set:

$$
X^{*}=\left(x 1^{*}, x 2^{*}, x 3^{*}, \ldots \ldots, x n^{*}\right)
$$

For bootstrap replication of $\theta \mathrm{e}$, equation (5) is considered:

$$
\theta_{b}^{*}=s\left(X_{b}^{*}\right), b=1,2,3, \ldots \ldots \ldots, B
$$

Where $X^{*}{ }_{b}$ is equal to the $\mathrm{b}^{\text {th }}$ bootstrap data set of $\mathrm{B}$ independent bootstrap data sets. The statistic of interest $(X)$ is the sample median $\sim x$ then $s\left(X^{*}\right)$ is the median of the bootstrap data set $\sim \chi^{*}$. Hence, B bootstrap replicates give an estimate of the $\theta \mathrm{e}$ distribution, which is the bootstrap estimate of the $2 \mathrm{G} / 3 \mathrm{G}$ average available bandwidth distribution, and its standard deviation is the bootstrap estimate of standard error for $\theta$ e given in [24]

$$
\begin{aligned}
& S E_{b}\left(\theta_{\theta}\right)=\sqrt{\frac{1}{B-1}} \sum_{b=1}^{B}\left(\theta_{b}^{*}-\epsilon,\right. \\
& \theta^{*}=\frac{1}{B} \sum_{b=1}^{B}
\end{aligned}
$$

When sampling is done with replacement, then it is possible that some $x_{i}$ would occur more than once or not at all. It can be mitigated with the smooth bootstrap technique where bootstrap data sets are constructed from a smooth empirical distribution ^ $F s$. The available bandwidth of the $2 \mathrm{G}$ and $3 \mathrm{G}$ access interfaces is computed by using equation (3-6).

Step 3 Compare available bandwidth of both the interfaces b1 (2G) \& b2 (3G) calculated with bootstrap approximation. Network having the highest available bandwidth is selected as access network for the real time multimedia application.

System under consideration for employing proposed network selection scheme is described in following section.

\section{Experimental Setup for Implementation of Proposed Algorithm}

We consider a heterogeneous environment consisting of GSM (2G) and HSDPA (3G) networks for network configuration as shown in Fig.2. Networks may be coupled in two ways-loosely-coupled and tightly coupled. In the loosely coupled scenario, two or more wireless networks are connected through internet [25]. In the tightly coupled case, $2 \mathrm{G}$ gateway is connected directly to the $3 \mathrm{G}$ gateway. $2 \mathrm{G}$ network appears to the router as another internal $3 \mathrm{G}$ router and thus becomes a part of the $3 \mathrm{G}$ network. In the present configuration, we consider loosely-coupled scenario. As $2 \mathrm{G}$ and $3 \mathrm{G}$ network have the comparable ability of supporting the service, it is decisive to keep the continuation and stability of application, when both networks are available simultaneously.

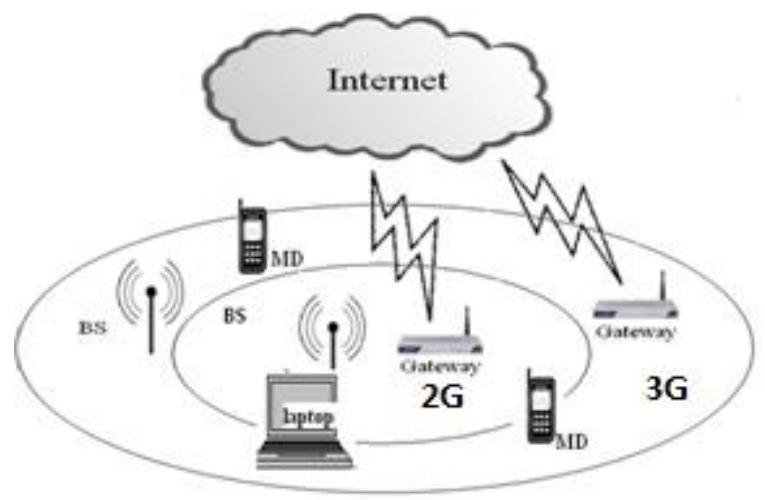

Fig 2: Heterogeneous wireless network model

$2 \mathrm{G}$ and $3 \mathrm{G}$ network based test bed is developed using dongles and net platform as shown in Fig 2 for practical implementation of proposed algorithm. It includes base stations and a multi mode mobile client like laptops, cell phones or PDAs. A hardware and software architecture module of dual mode client is shown in Fig 3.

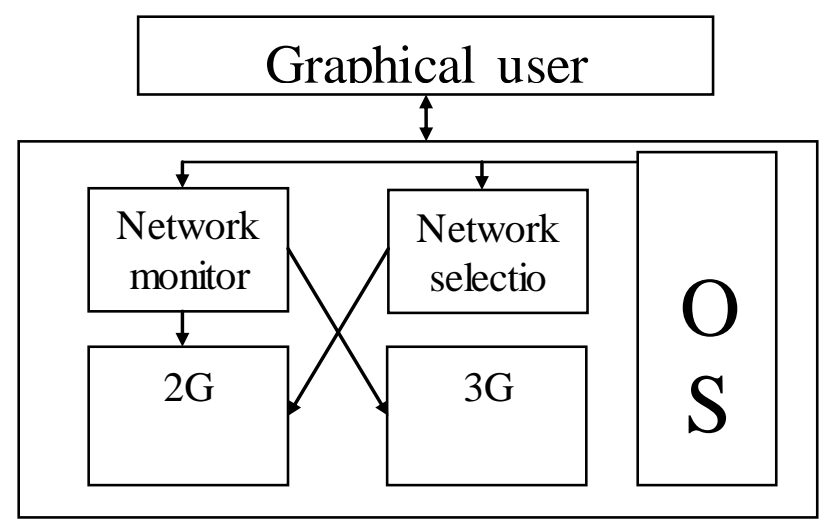

Fig. 3: (a) Software module of dual mode client

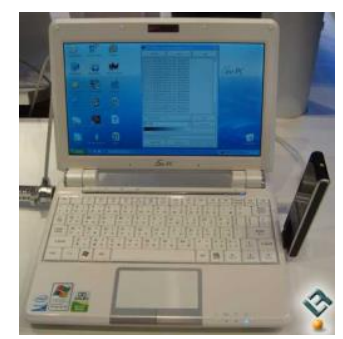

Fig. 3: (b) Hardware module of dual mode mobile client [26] 
Proposed algorithm's hardware implementation is supported by $2 \mathrm{G}$ and $3 \mathrm{G}$ dongle by huwaeii as interfaces for wireless networks. The software system includes modules to monitor, detect, decide and manage multiple wireless networks. Dual mode client hardware and software modules provide support for automatic roaming across $2 \mathrm{G}$ and $3 \mathrm{G}$ network. The client can monitor and automatically select $2 \mathrm{G}$ or $3 \mathrm{G}$ network according to the available bandwidth estimation technique. In accordance with the algorithm, interface of $2 \mathrm{G}$ was selected first and then $3 \mathrm{G}$.

\section{Results and Discussion}

Bootstrap approximation is a potential method for estimating available link bandwidth in wireless networks for network selection in wireless heterogeneous environment. For obtaining numerical results, we have performed the operation every second with sample size $n=5$ and number of bootstrap replications $B=10$. The number of bootstrap samples may be increased in accordance with the computing power available with the node. Increasing the number of samples can reduce the effects of random sampling errors.

The estimated available bandwidth at different instances with respective RSS of both interfaces is tabulated in Table 1. It is observed that the network with more available bandwidth is automatically selected for real time multimedia services. RSS is measured to see the presence various wireless network in region and contemporary status of the network in heterogeneous environment. It is also observed that available bandwidth is not exactly depended upon RSS. If RSS is large in value at any instance then it does not ensure that the respective network have high available bandwidth. Number of users accesses respective network at the instance of bandwidth estimation also prominently affected its value.

Table 1: Estimated available bandwidth with received signal strength in heterogeneous environment of $2 \mathrm{G}$ and $3 \mathrm{G}$ net works is tabulated

\begin{tabular}{|c|c|c|c|c|c|}
\hline S. No & \multicolumn{2}{|c|}{ 2G (GSM) } & \multicolumn{2}{c|}{ 3G (HSDPA) } & Network Selection \\
\hline & RSS $(\mathbf{d B m})$ & ABE $(\mathbf{K B})$ & RSS $(\mathbf{d B m})$ & ABE $($ KB $)$ & Selected network \\
\hline $\mathbf{1 .}$ & -76.0 & 38.79 & -75.0 & 29.83 & $2 \mathrm{G}$ \\
\hline $\mathbf{2 .}$ & -74.0 & 12.12 & -77.0 & 32.02 & $3 \mathrm{G}$ \\
\hline
\end{tabular}

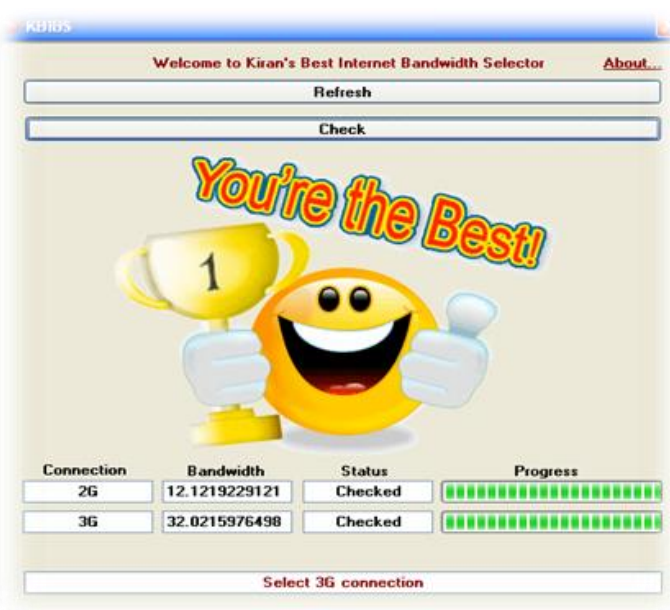

Fig 4: User interface of network selection algorithm based on available bandwidth estimation in heterogeneous environment of $2 \mathrm{G}$ and $3 \mathrm{G}$ network

The $2 \mathrm{G} / 3 \mathrm{G}$ network is selected for multimedia services as per user's inclination when comparing their available bandwidth based on bootstrap approximation, as shown in Fig 4.

Performance evaluation of proposed algorithm for network selection: For performance evaluation of the proposed algorithm following scenarios have been considered.

Case 1: In this case proposed algorithm is tested at different time and locations. It is observed from Fig. 5 (a) \& (b) that available bandwidth estimated at different times and locations are different because at any time or location the number of users share the wire less network is dynamic which leads to variation of the bandwidth available to a user. At any instance or location $2 \mathrm{G} / 3 \mathrm{G}$ can be selected depending upon their current status of ABW. It is measured in KB.

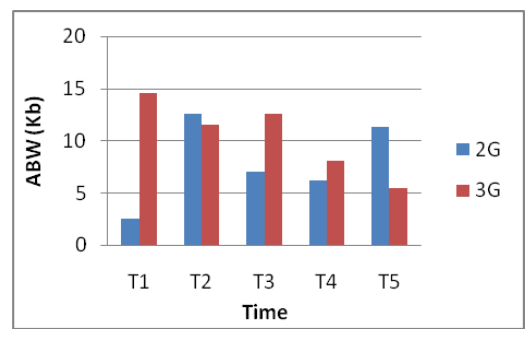

(a)

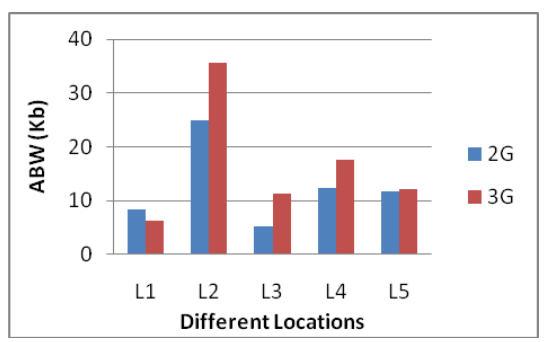

(b)

Fig. 5: (a) Represents available bandwidth vs. Time (b) Shows available bandwidth vs. different locations 
Case 2: In this case impact of downloading file size for bootstrap approximation to measure $\mathrm{ABW}$ is evaluated. The selection of size of file to be downloaded for estimation of real time available bandwidth by using bootstrap approximation is critical is sue. Choice of size of file to be downloaded depends upon the cost and time consumption bear by the active user. If selected file size is too large then the cost and time consumption increased. Although, if selected file size is too small then accuracy of estimation will going to be reduced. From Fig 5 (c) it is clear that if file size is too small such as $9 \mathrm{~KB}$ then estimation of $\mathrm{ABW}$ is not feasible or proper, but as the file size increases it starts working properly. In Fig 5 (d) as the file size increases the estimation time is also increases respectively in both wireless networks available in heterogeneous environment. 2G/3G network's ABW estimation time can be changed because at any instance the number of user in any of the wireless network is random. Any of the networks in heterogeneous environment can be busy due to this speed of downloading file is going slow down and estimation time increases in same proportion.

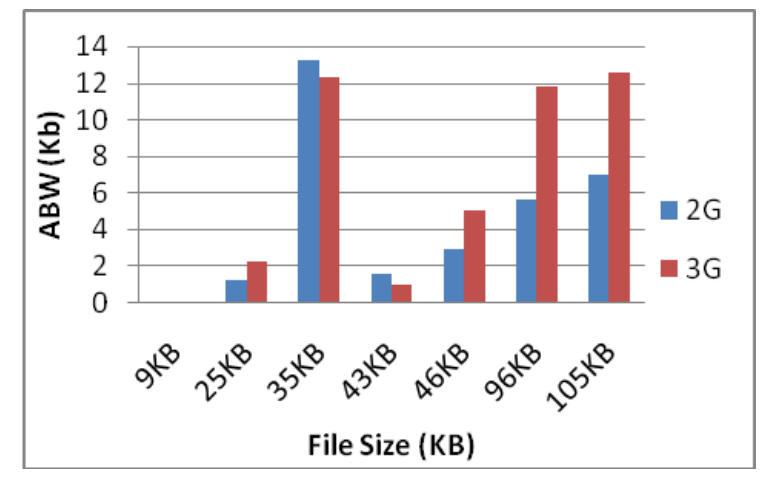

(c)

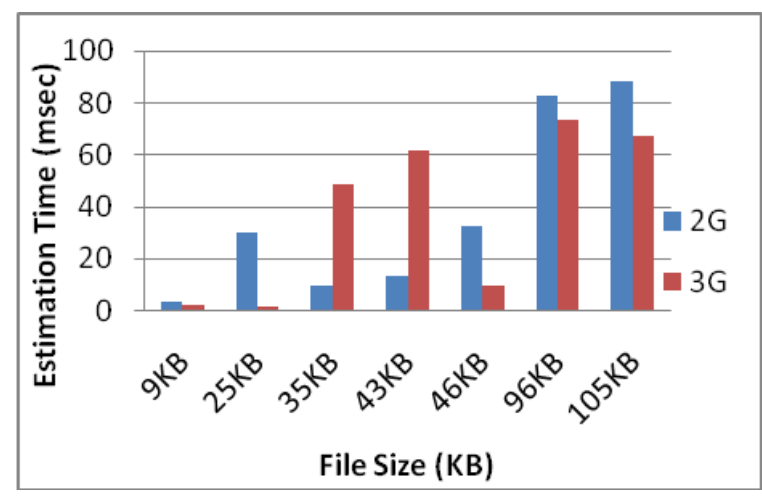

(d)

Fig. 5: (c) Represents file size vs. ABW (d) Shows file size vs. ABW estimation time

The results of the said algorithm are better in term of estimation time with the findings reported in [27] which further justify the validity of this algorithm.
Available bandwidth estimation with bootstrap approximation for network selection in heterogeneous environment is proved as a practical solution for seamless communication for multimedia services. Real time implementation and experimental results show the efficiency and effectiveness of proposed algorithm.

\section{Conclusion}

In this paper, a novel network selection algorith $\mathrm{m}$ proposed based on available link bandwidth estimation, which is one of the critical issues in heterogeneous wireless network environment for real-time multimedia communication. Available link bandwidth is estimated using bootstrap approximation method for network selection in heterogeneous environment of $2 \mathrm{G}$ and $3 \mathrm{G}$ networks. Bootstrapping estimates available bandwidth by taking averages, peaks, low points and standard deviation. It allows gathering many alternative versions of the single statistic that would ord inarily be calculated from one sample to raise accuracy and reliability of estimation. Performance evaluation of proposed network selection algorithm is carried out in both temporal and spatial domains in terms of estimation time and file size metrics to check its robustness. The proposed algorithm performs well in terms of accuracy and estimation time. The algorithm presented in this paper has potential to work in realistic environment of multi access networks

\section{References}

[1] Brusilovsky et al., "Selection of networks between WLAN and $2 \mathrm{G} / 3 \mathrm{G}$ networks based on user and provider preferences" Patent number US 7116970 B2, Oct 3, 2006.

[2] Alaeddine Gharsellaoui, Mohamed Khaled Chahine, "Optimizing Access point Selection in Wireless Local Area Network" International Conference on Communications and Information Technology (ICCIT), Aqaba, 29-31 March, 2011, pp 47-52

[3] Iqbal Hussain, Rabiullah Khattak, "Handover between 2G, 3G and WLANs", Electrical and Information Technology, Deptt. of Lund University, Study project by master student in wireless communication in 2009.

[4] Manish Jain, Constantinos Dovrolis, "End-to-End Available Bandwidth: Measurement Methodology, Dynamics, and Relation with TCP Throughput", IEEE/ACM Transactions On Networking, Vol. 11, No. 4, Aug 2003, pp. 537-549.

[5] D. Kiwior, J. Kingston, A. Spratt "PATHMON, A Methodology for Determining Available Bandwidth over an Unknown Network", IEEE/Sarnoff Sy mposium on Advances in Wired 
and Wireless Communication, 26th -27 th April 2004, pp. 27-30.

[6] Gu Wei-Xuan, Yu Shun-Zheng "Prioritized TriPackets Probes for Available Bandwidth Measurement", Proceedings of International Conference on Communications, Circuits and Systems, Guilin, China, 25th -28th June 2006, pp. $1777-1781$.

[7] Hyeong-Min Nam, Kyung-Seok Shin, Jae-Yun Jeong, Seong-Jun Bae, and Sung-Jea Ko "Probing Based Wireless Channel Bandwidth Estimation And Its Application To Video Streaming Over Wireless Networks", IEEE International Symposium on Consumer Electronics, Vilamoura, 14th -16th April 2008.

[8] Dawei XU, Depei QIAN “A Bandwidth Adaptive Method for Estimating End-to-End Available Bandwidth", 11th IEEE Singapore International Conference on Communication Systems, ICCS 2008, Guangzhou, China, 19th -21st Nov. 2008.

[9] Emanuele Goldoni, Giuseppe Rossi, Alberto Torelli "Assolo, a New Method for Available Bandwidth Estimation", Proceedings of Fourth International Conference on Internet Monitoring and Protection, ICIMP '09, Ven ice/Mestre, Italy, 24th -28th May 2009.

[10] Takashi Oshiba, Kazuaki Nakajima "Quick Endto-End Available Bandwidth Estimation for QoS of Real-Time Multimedia Communication", IEEE Symposium on Computers and Communications (ISCC), 2010, Riccione, Italy, 22nd -25th June 2010, pp 162 .

[11] Jorg Liebeherr, Markus Fidler, Shahrokh Valaee "A System-Theoretic Approach to Bandwidth Estimation" IEEE/ACM Transactions on Networking, Vol. 18, No. 4, Aug 2010, pp. 1040 1053.

[12] Anfu Zhou, Min Liu, Yilin Song, Zhongcheng Li, Hui Deng, Yuanchen Ma "A New Method for Endto-end Available Bandwidth Estimation", Proceedings of Global Telecommunications Conference, IEEE Globecom 2008, New Orleans, Nov. 30-Dec. 4 2008, LO.

[13] Yu-Chen Huang, Chun-Shien Lu, Hsiao-Kuang $\mathrm{Wu}$ "Available Bandwidth Estimation via OneWay Delay Jitter and Queuing Delay Propagation Model", Proceedings of Wireless Communications and Networking Conference, WCNC2006, Las Vegas, NV, USA, 3-6 April 2006, VOL. 1.

[14] Mehmet U. Demirc in, Peter van Beek "Bandwidth Estimation and Robust Video Streaming Over 802.11e Wireless LANs", Proceedings of IEEE International Conference on Multimed ia and Expo, ICME 2005, Amsterdam, Netherlands 6-6 July 2005.
[15] Xiliang Liu, Kaliappa Ravindran, Dmitri Loguinov, "A Queuing-Theoretic Foundation of Available Bandwidth Estimation: Single-Hop Analysis", IEEE/ACM Transactions on Networking, San Francisco, CA, USA, Vol. 15, No. 4, Aug 2007, pp. 918-931.

[16] Seung Yeob Nam, Sunggon Kim, Dan Keun Sung "Minimal Backlogging Method for Estimation of Available Bandwidth", IEEE Communications Society, Globecom 2004, pp. 1469-1473.

[17] Yu-Chen Huang1, Chun-Shien Lu1, Hsiao-Kuang $\mathrm{Wu}$, "Reliable Available Bandwidth Estimation Based on Distinguishing Queuing Regions and Resolving False Estimations" IEEE Communications Society, Globecom 2004, pp 4081-4086.

[18] Xiaojun Hei, Brahim Bensaou, Danny H.K. Tsang, "A light-weight available bandwidth inference methodology in a queuing analysis approach", Proceedings of IEEE International Conference on Communications, 16th - 20th May 2005.

[19] Yu Cheng, Vikram Ravindran, Alberto LeonGarcia, Hsiao-Hwa Chen, "New Exploration of Packet-Pair Probing for Available Bandwidth Estimation and Traffic Characterization", Proceedings of IEEE International Conference on Communications, 24th -28th June 2007.

[20] Kwangjin Choi, Seong Gon Choi, Jun-kyun Choi, Chinchol Kim, "Available Bandwidth Estimation with Consideration of Broadband Convergence Networks", Proceedings of 9th International Conference on Advanced Communication Technology, Glasgow, UK, 12th -14th Feb. 2007.

[21] Kiran Ahuja, Rajesh Khanna, Brahmjit Singh, "Real Time Available-Bandwidth Estimation (ABE) Algorithm Based Selection in Heterogeneous Network for W iMAX and 3G', in the proceeding of NGMAST 2011, Card iff Wales, U.K

[22] Mussa Bshara, Umut Orguner, "Fingerprinting Localization in Wireless Networks Based on Received-Signal-Strength Measurements: A Case Study on WiMAX Networks" IEEE Transactions On Vehicular Technology, VOL. 59, NO. 1, pp 283-294, January 2010

[23] Jacob Strauss, Dina Katabi, Frans Kaashoek, "A Measurement Study of Available Bandwidth Estimation Tools" IMC'03, October 27-29, 2003

[24] B. Efron and R. J. Tibshirani, "An Introduction to the Bootstrap", Chapman \& Hall, 1993.

[25] Daehyon Kim and Ganz, A., "Architecture for 3G and 802.16 wireless networks integration with QoS support". Second International Conference on Quality of Service in Heterogeneous Wired/Wireless Networks, Aug. 2005, pp.8. 
[26] http://www.legitrev iews.co m/images/news/2008/ee epc_wimax_dongle.jpg

[27] A.B. Downey, "Using pathchar to estimate Internet link chara J. U. Duncombe, "Infrared navigation Part I: An assessment of feasibility (Periodical style)," IEEE Trans. Electron Devices, vol. ED-11, pp. 3439, Jan. 1959. [On line]. Available: http://dx.doi.org/10.4316/ieee.1959.3422

\section{Authors' Profiles}

Kiran Ahuja has done outstanding research in the fields of optical and wireless communication. She received her B.Tech \& M.Tech degree in Electronics and Communication Engineering from Punjab Technical University, Jalandhar, India and pursuing Ph. $\mathrm{D}$ in Electronics \& Communication Engineering in wireless communication and networks field. She worked as faculty at DA VIET, Jalandhar.

Brahmjit Singh received B.E. degree in Electronics Engineering from Malaviya National Institute of Technology, Jaipur in 1988, M.E. degree in Electronics and Communication Engineering from Indian Institute of Technology, Roorkee in 1995 and $\mathrm{PhD}$ degree from Guru Gobind Singh Indraprastha University, Delhi in 2005 (INDIA). He started his career as a lecturer at Bundelkhand Institute of Eng ineering and Technology, Jhansi (INDIA). Currently, he is Dean \& Professor in School of ICT at Gautam Budhha University, Noida (INDIA). He was Ex-head of CCN \& ECE Department at National Institute of Technology, Kurukshetra (INDIA). He teaches post-graduate and graduate level courses on Wireless communication and CDMA systems. His research interests include mobility management in cellular / wireless networks, Planning, Designing and optimization of Cellular Networks \& Wireless network security. He has published 47 research papers in International/ National journals and Conferences. Dr. Brahmjit Singh received the Best Research Paper Award from 'The Institution of Engineers (India)' in 2006.

Rajesh Khanna was born in Ambala, India. He received B.Sc. (Engg.) Degree in Electronics \& Communication in 1988 from Regional Engineering College, Kurkshetra and M.E. degree in 1998 from Indian Institute of Sciences, Bangalore. He was with Hartron R\&D centre till 1993. Until 1999, he was in All India Radio as Assistant Station Engineer. Presently he is working as Associate Professor in the Department of Electronics \& Communication at Thapar Institute of Engineering \& Technology, Patiala. He has published 12 papers in national and International conferences. He has worth Rs 50 Lakhs projects to his credits.
How to cite this paper: Kiran Ahuja, Brahmjit Singh, Rajesh Khanna,"Available Link Bandwidth Based Network Selection in Multi-access Networks", International Journal of Intelligent Systems and Applications(IJISA), vol.6, no.3, pp.76-83, 2014. DOI: $10.5815 /$ ijisa.2014.03.08 